\title{
I-1 Transcription Regulation of Silk Genes
}

\author{
Yoshiaki Suzuki, Masaaki Tsuda, Yoshihide Tsujimoto \\ and Susumu Hirose \\ Laboratory of Cell Differentiation, National Institute for \\ Basic Biology, Okazaki City 444, Japan
}

\section{Developmental expression of fibroin and sericin genes}

Silk fibroin mRNA was isolated in a pure form from the posterior silk gland of Bombyx mori larva, and identified by its unique nature (Suzuki and Brown, 1972). Its size is about $16 \mathrm{~kb}$ (Lizardi and Brown, Cold Spring Habor Symp. Quant. Biol. $38,701,1973$ ), and its repetitious sequence, composed of about $40 \% \mathrm{G}+20 \% \mathrm{C}$, has been characterized by partial sequence analysis of the mRNA (Suzuki and Brown, 1972). The fibroin gene was detected by hybridization of the purified fibroin mRNA to genomic DNA (Suzuki, Gage and Brown, 1972). Concentration of the gene is $0.004 \%$ of the $B$. mori genome in any tissue, demonstrating that specific gene amplification does not take place even in tissue in which the gene is actively expressed (Suzuki, Gage and Brown, 1972); this concentration is equivalent to 1 copy of the gene per haploid complement (Gage and Manning, J. Mol. Biol. 101, 327, 1976).

The fibroin gene is transcribed specifically in the posterior silk gland, not in the middle silk gland or any other tissues (Suzuki and Brown, 1972; Suzuki and Suzuki, 1974; Suzuki and Giza, 1976; Tsuda, Ohshima and Suzuki, 1979; Maekawa and Suzuki, 1980) and at the third, fourth and fifth larval feeding stages of silkworm development, but not in the third and fourth molting stages (Suzuki and Suzuki, 1974; Maekawa and Suzuki, 1980).

Sericins are a group of several proteins which are synthesized specifically in the middle silk gland (Sprague, Biochemistry 14, 925, 1975; Gamo, Inokuchi and Laufer, Insect Biochem. 7, 7258, 1977); they serve as glue for fibroin fiber in construction of a cocoon. Abundant sericin mRNAs of $11 \mathrm{~kb}$ and $9.6 \mathrm{~kb}$ have been purified from the middle silk gland (Okamoto, Ishikawa and Suzuki, 1982). With the $11 \mathrm{~kb}$ mRNA used as a probe, the sericin gene clones were obtained and analyzed for their structure (Okamoto, Ishikawa and Suzuki, 1982). By Northern blotting of the middle silk gland RNA the gene for the $11 \mathrm{~kb}$ mRNA has been shown to be transcribed specifically in the fifth larval instar but not in earlier stages (Ishikawa and Suzuki, 1983). The $9.6 \mathrm{~kb}$ mRNA which shares a sequence homology to a great extent with the gene for $11 \mathrm{~kb}$ mRNA (Okamoto, Ishikawa and Suzuki, 1982) is transcribed only in the later stage of the fifth instar, not in the earlier stages. A gene which shares a sequence homology with the $5^{\prime}$ terminal region of the gene for $11 \mathrm{~kb}$ mRNA is transcribed in the third, fourth and fifth feeding stages but not in the third and fourth molting stages; in a manner very similar to fibroin gene transcription but in the middle silk gland (Ishikawa and Suzuki, 1983).

\section{Structural analysis of fibroin and sericin genes}

Fibroin genes have been cloned (Suzuki and Ohshima, 1977; Ohshima and Suzuki, 
1977) and analyzed for their structure (Tsujimoto and Suzuki, 1979a, 1979b; Tsuda, Ohshima and Suzuki, 1979). The structural part of the fibroin gene codes mainly for repetitious (Gly-Ala) $)_{n}$ sequences with an occasional and slight irregularity in the repetition. The fibroin gene is interrupted by a $970 \mathrm{bp}$ intron between nucleotide positions +67 and +1036 (Tsujimoto and Suzuki, 1979b). The $5^{\prime}$ end of a presumed precursor mRNA for fibroin was mapped on the fibroin DNA sequence and corresponded to the $5^{\prime}$ end position of fibroin mRNA (Tsuda, Ohshima and Suzuki, 1979); the position assigned was +1 . At nucleotide positions -30 to -24 a TATAAAA, a typical "TATA box" sequence was found (Tsujimoto and Suzuki, 1979a).

The sericin gene for the $11 \mathrm{~kb}$ mRNA is interrupted by at least several introns, and a part of the structural gene portion is composed of $114 \mathrm{bp}$ repetitious sequences which code for abundant (about $40 \%$ ) serine residues (Okamoto, Ishikawa and Suzuki 1982). The gene also contains a TATAAAA sequence at nucleotide positions -30 to -24 . A presumed transcription initiation site $(+1)$ was determined on the sericin DNA map, which was identical to the site corresponding to the $5^{\prime}$ end of the $11 \mathrm{~kb}$ mRNA (Okamoto, Ishikawa and Suzuki, 1982).

Comparing the $5^{\prime}$ flanking sequences of the fibroin and the sericin genes we have detected two regions having a high sequence homology between the genes; one region -209 to -179 of the fibroin gene is very homologous to -200 to -171 of the sericin gene, and the other -138 to -99 of the fibroin gene to -131 to -94 of the sericin gene (Okamoto, Ishikawa and Suzuki, 1982). The degree of homology is greater than $70 \%$ for both regions. Because such a high sequence homology of these regions can not be found in the $5^{\prime}$ flanking sequence regions of other genes, some biological role in relation to the regulation of the fibroin and sericin genes is expected (Suzuki, 1982).

A gross restriction map of the fibroin DNA from the posterior silk gland cells (producer cells) is indistinguishable from that from the tissue in which the fibroin gene is not expressed (nonproducer cells) (Manning and Gage, J. Biol. Chem. 253, 2044, 1978; Tsujimoto and Suzuki, 1979a). Sequence comparison between the cloned fibroin genes from the producer cells and the nonproducer cells, although the clones were derived from different strains of $B$. mori, indicated an identical sequence between -297 and +333 (Suzuki and Adachi, 1984): All the known transcription signals are localized in this region (Tsuda and Suzuki, 1983a).

To examine the methylation pattern on fibroin DNAs that comes from the producer cells and the nonproducer cells, we carried out restriction of the genomic DNAs with several restriction enzymes that recognize methylated bases from unmethylated ones. Southern blotting of these DNAs showed no difference between the producer and nonproducer cells in the region of -650 to +326 in the fibroin gene (Tsujimoto and Suzuki, 1984). As the number of sites tested by this method is very much limited, more extensive tests were desired. We have purified natural fibroin DNAs, without using a gene cloning procedure, from the posterior silk glands, the middle silk glands and the pupae (Tsujimoto and Suzuki, 1984). The exact purities of these preparations were not known, but they were greater than $15 \%$ pure. Upon gel electrophoresis and silver staining of the preparations a single DNA band of $17 \mathrm{~kb}$ Hind III-EcoRI fragment of the fibroin DNA was observed with no sign of contaminating DNAs. With these purified preparations DNA sequencing has been carried out by the method of Maxam and Gilbert. The DNA sequences at -171 to +102 of these preparations 
as well as those of the cloned genes were identical. Furthermore, on the sequencing gels there were no difference between the preparations and no sign of ${ }^{5 \mathrm{~m}} \mathrm{C},{ }^{3 \mathrm{~m}} \mathrm{~A}$ and ${ }^{7 \mathrm{~m}} \mathrm{G}$ (Tsujimoto and Suzuki, 1984). The template activities of these preparations tested in the cell-free system prepared from the middle silk glands were indistinguishable from each other and from the activity of the cloned fibroin gene (Tsujimoto and Suzuki, 1984). These results strongly suggest that the fibroin genes from the producer and nonproducer cells are identical in their primary sequences as well as in their base modifications.

\section{Transcription signals of fibroin and sericin genes}

A series of $5^{\prime}$ deletion mutants and $3^{\prime}$ deletion mutants of the fibroin gene were prepared, and their transcription activities were analyzed in in vitro transcription systems and an in vivo transcription system. Transcription of these mutants in a heterologous cell-free system, a HeLa cell extract prepared by the method of Manly et al. (Proc. Natl. Acad. Sci. USA 77, 3855, 1980), indicated that nucleotide sequences from -29 to +6 are sufficient to support an efficient and faithful transscription of the fibroin gene (Tsujimoto et al., 1981). Transcription in a homologous cell-free system, a posterior silk gland extract prepared in a manner similar to the HeLa cell extract, also indicated the same 35 nucleotide stretch as the basic promoter of the fibroin gene (Tsuda and Suzuki, 1981). In this homologous system, however, sequences upstream from the "TATA box" gave a 2-fold enhancement of transcription. This enhancement is discussed in detail below.

Efficient methods to produce single base substitution mutants of the fibroin gene have been developed that use small DNA fragments mutagenized with $\mathrm{NaNO}_{2}$ (Hirose, Takeuchi and Suzuki, 1982) or synthetic oligonucleotides of designed sequences (Hirose et al., 1984) as a primer. Transcription tests of these mutants in the HeLa cell extract and in the posterior silk gland extract indicated that nucleotides in the "TATA" box region and the region around -20 are important for promoter function (Hirose, Takeuchi and Suzuki, 1982).

Transcription tests of various mutants of the fibroin gene in COS- 1 cells, monkey kindney cells transformed with SV40 genome, revealed the importance of the "TATA" box region and the region near the transcription start site (Tokunaga, Hirose and Suzuki, 1984). Even in this living cell system sequences upstream from the "TATA" box were not important in supporting efficient and faithful transcription of the fibroin gene.

Cell-free transcription of the fibroin gene and its mutants in the homologous cell extracts indicated the importance of upstream sequences, about the $200 \mathrm{bp}$ stretch from the "TATA" box, in enhancing transcription. Under a competitive transcription condition, enhancement was about 10-fold the basic promoter having the "TATA" box (Tsuda and Suzuki, 1983a). The 200bp stretch may be divided into two regions; one at -238 to -116 and the other at -73 to -53 . The sequence element from -234 to -66 can still function in its inverted orientation. Deletion of $20 \mathrm{bp}$ sequence at -65 to -44 gives a reduction of enhancement but still maintains some enhancement. Insertion of a $275 \mathrm{bp}$ DNA fragment at the same site, however, abolishes the enhancement completely. Upstream sequences of the sericin gene for $11 \mathrm{~kb}$ mRNA also produce enhancement; exact delimitation has not been carried out but the signal is localized between -1400 and -50 . This element when placed in front of the "TATA" box region of the fibroin gene can function as an enhancer. The enhancer of the fibroin gene and the enhancer of the sericin gene compete with each other in the same 
transcription system. This is probably caused by the homologous sequences shared between the genes, as indicated above. The Ad-2 major late gene also shows enhancement in the HeLa cell extract, but not in the silk gland extracts. Therefore, the enhancer signals of the fibroin and sericin genes probably are recognized by factors present only in the homologous cell extract.

Factors that recognize the enhancers

It is not known whether these factors that recognize the enhancers of the fibroin and sericin genes are related to the regulatory expression of those genes. Partial purification of the factors have been initiated (Tsuda and Suzuki, 1983b) to characterize the nature of the factors. Characterization of these factors and structural and functional analyses of the chromatin of the fibroin and sericin genes may contribute to our understanding of the mechanisms of tissue specific regulation of these genes.

\section{REFERENCES}

Hirose, S., Takeuchi, K. and Suzuki, Y. (1982). Proc. Natl. Acad. Sci. USA 79, 7258-7262.

Hirose, S., Takeuchi, K., Hori, H., Hirose, T., Inayama, S. and Suzuki, Y. (1984). Proc. Natl. Acad. Sci. USA 81, 1394-1397.

Ishikawa, E. and Suzuki, Y. (1983). Abstracts of the sixth annual meeting of Jap. Soc. Mol. Biol. p. 61 .

Maekawa, H. and Suzuki, Y. (1980). Develop. Biol. 78, 394-406.

Ohshima, Y. and Suzuki, Y. (1977). Proc. Natl. Acad. Sci. USA 74, 5363-5367.

Окамото, H., Ishikawa, E. and Suzuki, Y. (1982). J. Biol. Chem. 257, 15192-15199.

Suzuki, Y. (1982). in Embryonic Development, Part A: Genetic Aspects, eds. Burger, M. M. and Weber, R., pp. 305-325., Alan R. Liss, New York.

Suzuki, Y. and AdAchi, S. (1984). Develop. Growth and Differ. 26, 139-147.

Suzuki, Y. and Brown, D. D. (1972). J. Mol. Biol. 63, 409-429.

Suzuki, Y., Gage, L. P. and Brown, D. D. (1972). J. Mol. Biol. 70, 637-649.

Suzuki, Y. and Giza, P. E. (1976). J. Mol. Biol. 107, 183-206.

Suzuki, Y. and Oнsнima, Y. (1977). Cold Spring Harbor Symp. Quant. Biol. 42, 947-957.

Suzuki, Y. and Suzuki, E. (1974). J. Mol. Biol. 88, 393-407.

Tokunaga, K., Hirose, S. and Suzuki, Y. (1984). Nucleic Acids Res. 12, in press.

Tsuda, M., Ohshima, Y. and Suzuki, Y. (1979). Proc. Natl. Acad. Sci. USA 76, 4872-4876.

TsudA, M. and SuzuKi, Y. (1981). Cell 27, 175-182.

Tsuda, M. and Suzuki, Y. (1983a). Proc. Natl. Acad. Sci. USA 80, 7442-7446.

Tsuda, M. and Suzuki, Y. (1983b). Abstracts of the sixth annual meeting of Jap. Soc. Mol. Biol. p. 76.

Tsujimoto, Y., Hirose, S., Tsuda, M. and Suzuki, Y. (1981). Proc. Natl. Acad. Sci. USA 78, 48384842.

Tsujimoto, Y. and Suzuki, Y. (1979a). Cell 16, 425-436.

Tsujimoto, Y. and Suzuki, Y. (1979b). Cell 18, 591-600.

Tsujimoto, Y. and Suzuki, Y. (1984). Proc. Natl. Acad. Sci. USA 81, 1644-1648. 